\title{
Fuel Monitoring in ITER Plasma with the use of Neutral Particle Analysis
}

\section{Petrov ${ }^{1}$}

Ioffe Institute

26 Polytekhnicheskaya st., St.Petersburg, 194021, Russia

E-mail: mpetrovanpd.ioffe.ru

\section{Afanasyev}

Ioffe Institute

26 Polytekhnicheskaya st., St.Petersburg, 194021, Russia

E-mail: valenpd.ioffe.ru

\section{Mironov}

Ioffe Institute

26 Polytekhnicheskaya st., St.Petersburg, 194021, Russia

E-mail: maximenpd.ioffe.ru

\section{Nesenevich}

Ioffe Institute

26 Polytekhnicheskaya st., St.Petersburg, 194021, Russia

E-mail: vnesenevichenpa.ioffe.ru

\section{P. Goncharov}

St.Petersburg Polytechnic University

29 Polytekhnicheskaya st., St.Petersburg, 195251, Russia

E-mail: goncharovaphtf.stu.neva.ru

\section{Sergeev}

St.Petersburg Polytechnic University

29 Polytekhnicheskaya st., St.Petersburg, 195251, Russia

E-mail: v.sergeevespbstu.ru

\section{Timokhin}

St.Petersburg Polytechnic University

29 Polytekhnicheskaya st., St.Petersburg, 195251, Russia

E-mail: v.timokhinespbstu.ru

First EPs Conference on Plasma Diagnostics - $1^{\text {st }}$ ECPD 14-17 April 2015,

Villa Mondragone, Frascati (Rome) Italy

${ }^{1}$ Speaker 


\section{Introduction}

The main goal of Neutral Particle Analysis (NPA), currently being developed at A.F. Ioffe Physical-Technical Institute (St. Petersburg, Russia) for ITER, is to measure the hydrogen isotope composition of plasma on the basis of measurements of neutralized fluxes of corresponding hydrogen ions [1]. NPA diagnostic is included in the list of ITER first priority diagnostics as a part of ITER control system intended to provide the optimal D/T ratio in the plasma and to get the most effective plasma burning. Other tasks are to measure the distribution functions of thermal ions and also of fast (including alpha particles) ions, which result due to additional heating and nuclear fusion reactions.

The tandem of two neutral particle analyzers - Low Energy NPA (LENPA) and High Energy NPA (HENPA) - will be used on ITER. The analyzers are situated one behind the other and connected to the vacuum vessel via single vacuum tube, located close to the torus midplane along the main radius. Their lines of sight are independent inside the tube, so the analyzers can measure the atomic fluxes simultaneously. LENPA provides the measurement of $\mathrm{D}^{0}$ and $\mathrm{T}^{0}$ atoms within the energy range 10-200 keV. HENPA serves to measure $\mathrm{D}^{0}$ atoms in the energy range $0.16-2.2 \mathrm{MeV}$ and $\mathrm{T}^{0}$ atoms in the range $0.1-1.5 \mathrm{MeV}$. Both analyzers use thin carbon foils (thickness $\sim 100 \AA$ ) for the stripping of incoming atoms and similar dispersion systems of EllB type for the energy and mass analysis.

In this paper we study the effect of D/T pellet injection on NPA experimental data in Inductive ITER scenario under different assumptions: a) different transport models and b) different outward horizontal drift of the ablated pellet material. The sensitivity of NPA signals to these assumptions is estimated. The temporal behaviour of NPA count rates for different values of horizontal drift was modeled. The influence of ITER heating and diagnostic beams on the NPA D/T measurements is also studied and the results of the modeling are discussed.

\section{Connection between ITER transport models and NPA signal}

Two transport models have been taken into consideration: the empirical scale based model ITER-98(y,2) [2] and the gyro-Landau-fluid GLF23 theory based model [3]. A comparison between these approaches has been done from the point of view of NPA measurements. ASTRA code [4] has been run for the calculation of temperature and density profiles. Fig. 1 shows that ITER-98 transport model predicts 'flat' density profiles. In turn, temperature and density profiles given by GLF23 model are more peaked (Fig.2), the fuel ion density is higher, and the temperature is lower than in the case of ITER-98 model.

On average, GLF23 model gives NPA counting rates that are lower than the corresponding values in the case of ITER-98 model. This fact can be explained by the higher density and the lower temperature of the plasma predicted by GLF23 model. The denser plasma leads to weaker penetration of the wall neutrals into the plasma and to higher attenuation of the outgoing neutral fluxes at thermal energies. And the lower temperature means fewer ions with a high energy.

Characteristic $1 \mathrm{kHz}$ level shown in Figs 3 and 4 indicates the statistics value, which means $10 \%$ of accuracy of the neutral particle flux measurement within $0.1 \mathrm{~s}$ time window. 


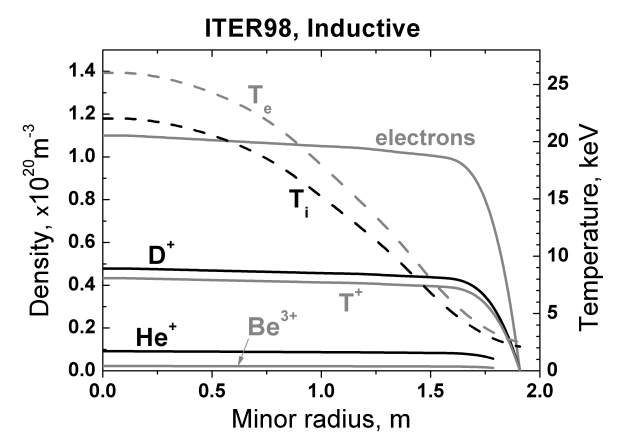

Fig. 1 Temperature and density profiles for ITER-98(y,2) scaling model

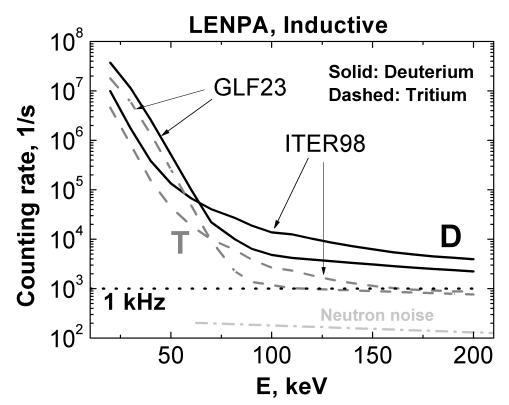

Fig.3 LENPA count rates for ITER-98(y,2) scaling and GLF23 models

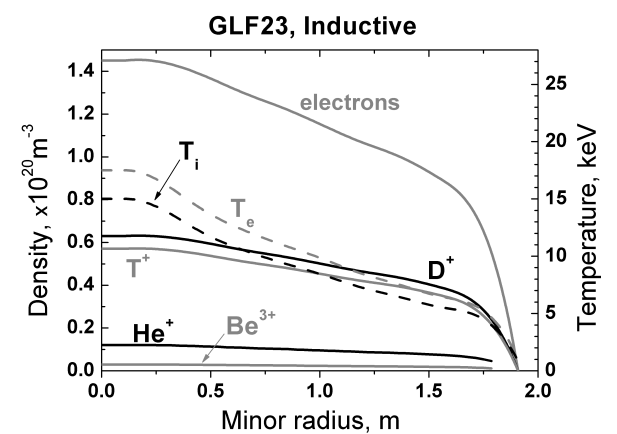

Fig. 2 Temperature and density profiles for GLF23 model

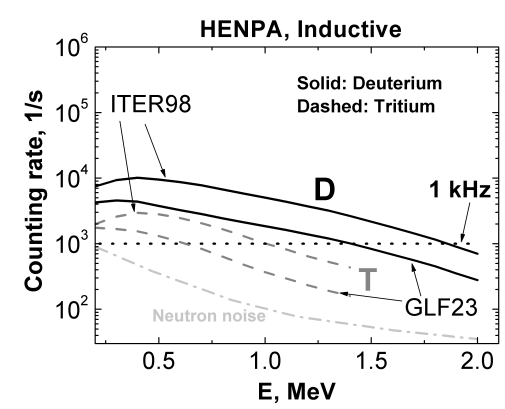

Fig.4 HENPA count rates for ITER-98(y,2) scaling and GLF23 models

It seems that the comparison of ITER NPA experimental count rates and NPA count rates modeled with the use of different density and temperature profiles provides an opportunity to determine what model - ITER98 or GLF23 - is more close to reality.

\section{Pellet radial drift and fuel deposition}

ITER plasma refueling will be provided by $\mathrm{D}, \mathrm{T}$ pellet injection. In a toroidal magnetic field the pellet ablation cloud become polarized ( $\nabla \mathrm{B}$-drift) and drifts across the field lines in the outward direction $(\mathrm{E} \times \mathrm{B}$-drift) [5,6]. The pellet deposition profile and the plasma fueling efficiency strongly depend on the expected pellet drift. Three cases of deposition profiles with $0 \%, 50 \%$, and $100 \%$ drift of a 50/50 DT-pellet injected from the high field side (HFS) of ITER plasma at pellet velocity $300 \mathrm{~m} / \mathrm{s}$ [6] are shown in Fig.5.

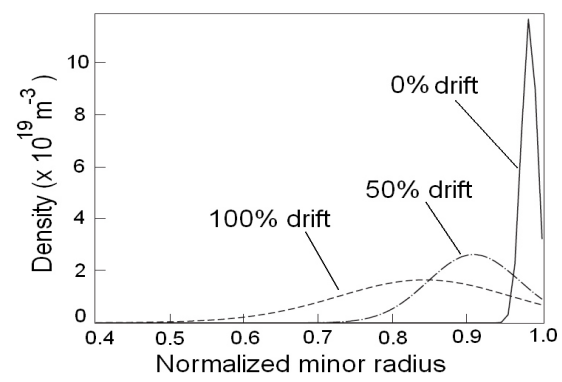

Fig.5 Pellet deposition profiles for three cases of 0\%, 50\%, and 100\% calculated pellet drift [7] 


\section{Plasma profiles evolution after pellet injection}

The ion density evolution in ITER during the pellet injection has been studied. It has been shown that NPA diagnostics can provide data on the time evolution of the ion density during ITER fueling cycle. In the modeling the GLF23 transport model has been used. It has been assumed that a 50/50 DT-pellet (cubic shape, $5 \mathrm{~mm}^{3}$ ) is injected at $\mathrm{v}=300 \mathrm{~m} / \mathrm{s}$ from the HFS of the plasma. The case of 50\% pellet drift has been considered. At the moment of pellet injection the edge density rises, while the temperature drops. The evolution of plasma profiles is demonstrated by two time slices: $\mathrm{t}=0 \mathrm{~s}$ (Fig.6) and $\mathrm{t}=0.2 \mathrm{~s}$ (Fig.7). The profiles restore after $0.4 \mathrm{~s}$ time interval.

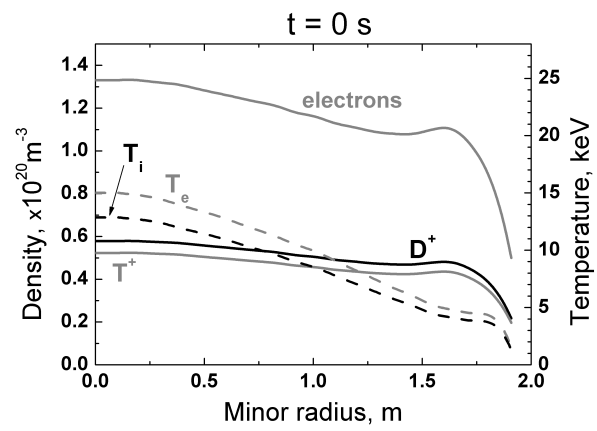

Fig.6 Plasma density and temperature at the moment of pellet injection

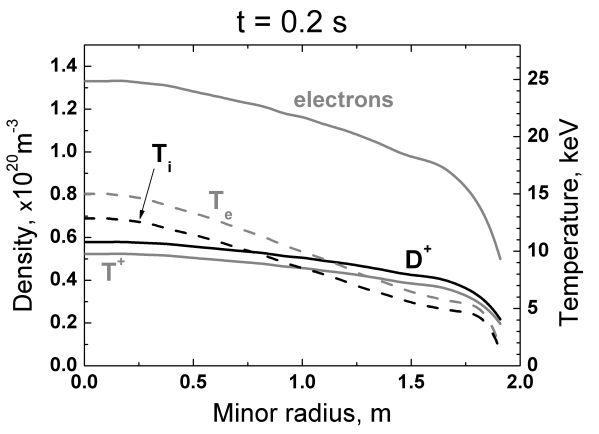

Fig.7 Plasma density and temperature $0.2 \mathrm{~s}$ after pellet injection

\section{NPA counting rate after pellet injection}

The emissivity function is proportional to the total probability for the atom of a given energy to be born at a certain location and successfully escape from the plasma volume. The analysis of the emissivity function for LENPA energy range shows [1] that atoms with energies of approximately $50 \mathrm{keV}$ represent the layer around $\mathrm{r} / \mathrm{a} \sim 0.7 \div 0.9$ deep in the plasma. More energetic $100 \mathrm{keV}$ atoms come mainly from $\mathrm{r} / \mathrm{a} \sim 0.4 \div 0.7$ layer. Such behavior is caused by a gradient of the temperature profile. The emissivity profiles for the most atom energies measured by HENPA have their maxima close to the plasma center. In turn, the pellet deposition profile is concentrated in the outer region of the plasma. Thus only the low energy analyzer LENPA can be used to provide information on the pellet drift effect and the time evolution of the ion density perturbation caused by the pellet injection.

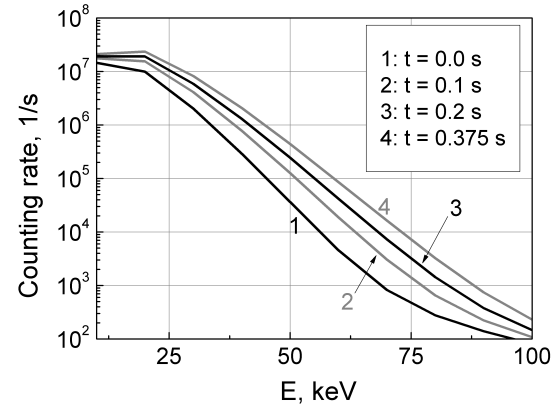

Fig.8 LENPA tritium count rates related to different moments after pellet injection. $50 \%$ drift is assumed.

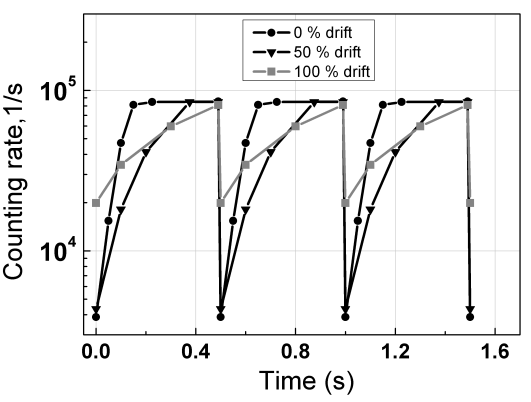

Fig. 9 Time dependence of LENPA tritium signal at the energy $\mathrm{E}=60 \mathrm{keV}$ during pellet injection with frequency $2 \mathrm{~Hz}$ 
Fig. 8 shows the evolution in time of LENPA energy spectra after the pellet injection. In calculations a 50/50 DT-pellet (cubic shape, $5 \mathrm{~mm}^{3}$ ) injected from the HFS of the plasma has been considered, assuming $300 \mathrm{~m} / \mathrm{s}$ pellet velocity and $2 \mathrm{~Hz}$ injection frequency. Fig. 9 presents the time dependence of LENPA tritium signal at the energy $\mathrm{E}=60 \mathrm{keV}$ for different values of horizontal drift. This plot shows that analysis of temporal behavior of NPA count rates can allow us to get the estimate of the drift.

\section{ITER neutral beam injection system and effect of NBI on NPA counting rate}

ITER Neutral Beam system is shown in Fig.10. Two heating injectors provide $1 \mathrm{MeV}$ deuterium Heating Neutral Beams (HNB) with the total injection power of $16.5 \times 2=33 \mathrm{MW}$. Diagnostic Neutral Beam (DNB) injects $100 \mathrm{keV}$ hydrogen atoms with equivalent current $\geq 20$ A [8].

The influence of the neutral beam injection (NBI) on the fast ion distributions in ITER has been investigated in the view of NPA diagnostic measurements. Calculations of the distribution functions of fast ions resulting from NBI have been performed. Corresponding neutralized fast ion fluxes and NPA counting rates have been calculated.

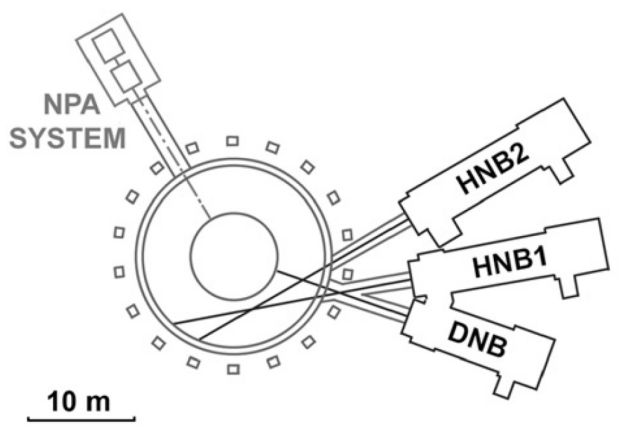

Fig.10 ITER Neutral Beam system

NPA performance analysis has been carried out and the operating limits of the NPA diagnostic system have been amended. In Fig.11 and Fig.12 the calculated NPA signals are presented for the case of Inductive ITER scenario.

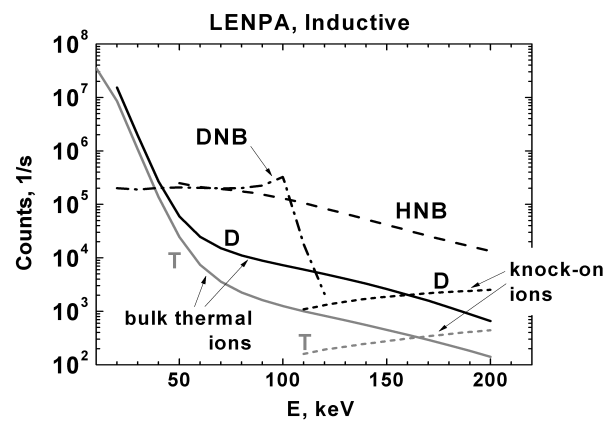

Fig. 11 LENPA signals in the presence of $\mathrm{HNB}$ and DNB

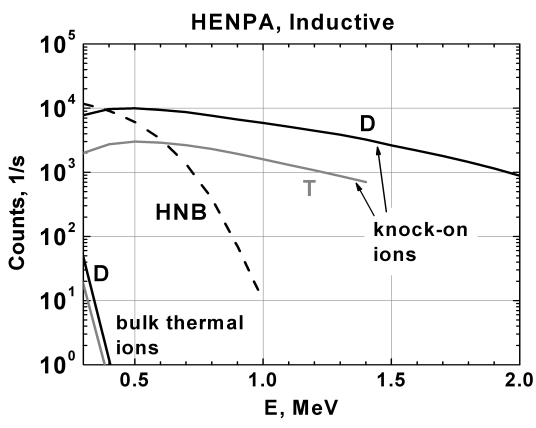

Fig.12 HENPA signals in the presence of HNB and DNB 
Contributions from different ion populations are shown apart from each other: bulk thermal ions, HNB and DNB ions, and knock-on ions. Fast knock-on D and T ion populations are the result of the close elastic collisions between thermal fuel ions and fusion $\alpha$-particles. It can be seen that in the thermal energy range $\mathrm{E}<200 \mathrm{keV}$ the neutralized $\mathrm{D}^{0}$ flux from the slowing down heating NB ions prevails over the signal from bulk thermal D ions down to energies $\mathrm{E} \sim 40 \mathrm{keV}$. In the $\mathrm{MeV}$ energy range the measurements are not distorted by NBI for the energies $\mathrm{E}>0.4$ $\mathrm{MeV}$, thus this energy range can be used for the analysis of fast knock-on $\mathrm{D}^{0}$ fluxes.

\section{Conclusions}

Our studies of D/T pellet injection in ITER Inductive discharges show that NPA signal is sensitive enough to determine what of the two transport models - ITER98 or GLF23 - is more close to reality and to estimate the value of outward horizontal drift of the ablated pellet material.

It has been shown that ITER heating beams have significant influence on the NPA D/T measurements. However, the $\mathrm{E}<40 \mathrm{keV}$ and $\mathrm{E}>400 \mathrm{keV}$ energy ranges are not affected by the beam injection and can be used for $\mathrm{D} / \mathrm{T}$ analysis.

The work was partially supported by Institution "Project Center ITER" under Russian Federation Contracts No. 02-14/01, No. 03-14/01.

\section{References}

[1] Afanasyev V A et.al., Neutral Particle analysis on ITER: present status and prospects, Nucl. Instr. and Meth. A 621 456-467 (2010)

[2] ITER Physics Expert Groups on Confinement and Transport and Confinement Modelling and Database, ITER Physics Basis Editors and ITER EDA, Nucl. Fusion 392175 (1999)

[3] R. E. Waltz et. al., A gyro-Landau-fluid transport model, Phys. Plasmas 42482 (1997)

[4] Pereverzev G.V. et.al., ASTRA: Automated System for Transport Analysis in Tokamak, Max-Planck Institutes Report IPP 5/98 (1998)

[5] V. Rozhansky et. al., Mass deposition after pellet injection into a tokamak, Plasma Phys. Control. Fusion 46 575-591 (2004)

[6] B. Pégouríe, et.al., Homogenization of the pellet ablated material in tokamaks taking into account the DB-induced drift, Nucl. Fusion 47 44-56 (2007)

[7] V. Parail et. al., Integrated modelling of ITER reference scenarios, Nucl. Fusion 49075030 (2009)

[8] ITER Design Description Document (DDD 5.3) Neutral Beam Heating \& Current Drive (NB $H \& C D)$ System, ITER Report N 53 DDD 29 01-07-03R 0.1 